\title{
Testing gustatory function using either a forced-choice or a non-forced-choice paradigm - Does it make a difference?*
}

\author{
Gerold Besser ${ }^{1}$, Alexander Prass| ${ }^{2}$, Christian A. Mueller', Bertold Renner ${ }^{3}$ \\ Department of Otorhinolaryngology and Head and Neck Surgery, Medical University of Vienna, Austria \\ 2 Department of Anaesthesiology, Medical University of Vienna, Austria \\ ${ }^{3}$ Institute of Experimental and Clinical Pharmacology and Toxicology, University of Erlangen-Nürnberg, Erlangen, Germany
}

Rhinology 57: 0, 000 - 000, 2019

https://doi.org/10.4193/Rhin19.021

*Received for publication:

January 22, 2019

Accepted: April 2, 2019

\begin{abstract}
Background: Recently more interest is evolving for research on gustatory function, also due to findings of "extraoral" taste receptors with postulated participation in a pathogen detection network. Also, bitter taste function seems to be reduced in patients with chronic rhinosinusitis. For testing gustatory function "taste strips" (TS) have been validated in a forced-choice (fc) and a non-forced-choice (nfc) paradigm and used in several studies. Purpose of the investigation was to evaluate possible differences of named modalities.
\end{abstract}

Methodology: Healthy subjects ( $n=102)$ with subjective normal gustatory function and patients $(n=30)$ with potential taste dysfunction were included. All participants were tested twice (using TS in four concentrations of sweet, sour, salty, and bitter taste), either starting with a fc or a nfc paradigm.

Results: In tested patients there was a difference between $\mathrm{fc}$ and $\mathrm{nfc}$ procedure with higher results for bitter in the fc testing procedure, while other qualities did not differ. This effect was also visible in the overall participants with higher scores in the fc procedure for bitter taste.

Conclusion: TS are valid to be used in a forced and a non-forced choice paradigm, with bitter taste showing slightly higher scores in forced-choice testing. Future investigators with focus on bitter taste perception should be particularly cautious when comparing results in regard to testing procedure.

Key words: bitter taste, chorda tympani nerve, gustation, psychophysics, taste strips

\section{Introduction}

Visual and olfactory stimuli precede taste detection of nourishments. This already creates a vague idea of taste prior to food ingestion and evaluates food safety ${ }^{(1)}$. If intake decision is made, hence food is thought to be safe, taste perception may detect toxic compounds (predominantly as bitter perception), and can, even unconsciously initiate food rejection. Taste receptors, located on the tongue and on the soft palate, are able to detect salty, sweet, sour, bitter and umami taste ${ }^{(2)}$. Retronasal olfactory function and trigeminal perception (mediating temperature and texture) complete food distinguishing and flavour perception. Interactions of these functions strongly affect dietary behaviour (3-5).
Possible health related consequences of disturbed flavour perception and misguided food selection are issues of ongoing research. Still, even though quite frequent, impairments of olfactory function, and in consequence flavour perception, are often overseen in clinical routine and handled poorly by physicians ${ }^{(6)}$. The taste system alone however is very resistant to damage and capable of regeneration. Ageusia hence is a very rare condition (2).

Patients reporting no impairment in taste function do usually not suffer from taste dysfunction ${ }^{(7)}$. On the other hand, patients suffering from disturbances of smell function, report both smell and taste dysfunction prior to diagnosis ${ }^{(4)}$. This is most likely due to the mentioned important and misinterpreted retronasal 
olfactory function. "Taste" perception is believed to be decreased, whereas retronasal olfaction (and in consequence flavour perception) is essentially impaired, but the five qualities of taste receptors are still detected ${ }^{\left({ }^{(8)}\right.}$. Furthermore, in patients with impaired olfactory function, also taste sensitivity may be reduced $^{(9)}$. These circumstances underline the necessity of separate olfactory and gustatory testing ${ }^{(10,11)}$.

Recently more interest is evolving for research on gustatory function, also in consequence of findings identifying bitter and sweet taste receptor expression beyond the tongue ${ }^{(12)}$. So called "extraoral" taste receptors can also be found in motile cilia of the airway epithelium and are postulated to be part of a pathogen detection network ${ }^{(13)}$. In consequence taste receptor malfunction might play a role in inflammatory processes, such as chronic rhinosinusitis (CRS) ${ }^{(13,14)}$. Distinct polymorphisms of bitter taste receptor genotypes may predispose individuals to bacterial growth in the sinuses ${ }^{(15)}$. Furthermore, in CRS patients oral bitter taste detection seems to be impaired ${ }^{(16,17)}$. Hence research in the field of taste perception appears of high value in order to understand pathomechanism of various diseases and maybe enlighten possible targets for new therapy options.

For gustatory testing "whole mouth" and "regional" tests are available. "Taste strips", as a whole mouth and a regional testing method, have been shown to be reliable, easy to administer and have the advantage of a long shelf life. Taste strips can also be self-administered and consequently safe time and personnel (18). The test consists of 16 spoon-shaped impregnated filter paper strips and two blanks that are presented to patients in a pseudorandomized sequence.

Taste strips have initially been validated in a non-forced-choice (nfc) manner (by providing the possibility to answer "no taste") for diagnosing of taste dysfunction ${ }^{(8)}$ and have been used in this manner in several studies ${ }^{(16,19-22)}$. Additionally, an extended version of the taste strips has been validated, providing further data on the non-forced-choice paradigm ${ }^{(23)}$. In a series of other studies, the taste strips test was administered with a forcedchoice ( $\mathrm{fc}$ ) paradigm ${ }^{(17,24-26)}$, including for the assessment of normative data ${ }^{(27)}$.

The effect on taste strips test results in regard to the applied paradigm has not been investigated in a direct comparison so far. Purpose of the following investigation was to evaluate differences in results using the taste strips in a forced-choice and non-forced-choice paradigm.

\section{Materials and methods}

Firstly, voluntary healthy participants were recruited through invitational notices displayed in the area of the Medical University of Vienna. Secondly, patients undergoing surgeries with possible negative effects on taste function and patients reporting taste dysfunction were included. The protocol was approved by the local ethics committee (EK Nr. 1548/2013) and was conducted according to the guidelines of the declaration of Helsinki on biomedical research involving human subjects. Subjects and patients provided their written informed consent.

\section{Healthy participants}

A total of 102 healthy subjects ( 51 female, 51 male; mean age \pm standard deviation (SD), $38.3 \pm 18.1$ years) were included. Only subjects with subjective normal gustatory function were included as healthy participants. Subjective assessment of taste (SAT), as well as smell function (SAS) and current state of hunger $(\mathrm{SAH})$ were evaluated on a ten-point scale (1=no taste/smell/ hunger, $10=$ excellent taste/smell/hunger) before psychophysical testing. Tongue and oral cavity were examined to rule out taste impairing diseases (e.g., infections, thrush, ulcer). Also, history for head surgeries, medication intake, smoking history, mouth burning, mouth dryness, and body measurements were recorded as possible confounding factors.

Patients with potential taste dysfunction

Thirty patients ( 19 female, 11 male; mean age \pm SD, $41.9 \pm 16.7$ years) who either reported taste dysfunction or were operated in areas which might affect taste function, were additionally included.

\section{Gustatory testing}

Participants had to refrain from eating, drinking and smoking at least one hour prior to testing. All participants were randomized and tested twice, with a short interval in between, in a crossover design, either starting with a forced or a non-forced choice paradigm. Testing duration was 10 to 15 minutes in average for one test cycle. Breaks in between cycles were used to collect demographic data and subjective assessments. Each participant was tested with 18 taste strips (TS), pseudo-randomly administered. Sixteen filter paper strips were impregnated with four concentrations of sweet (0.4 / 0.2 / $0.1 / 0.05 \mathrm{~g} / \mathrm{ml}$ sucrose), sour ( $0.3 / 0.165 / 0.09 / 0.05 \mathrm{~g} / \mathrm{ml}$ citric acid), salty $(0.25 / 0.1 / 0.04$ $/ 0.016 \mathrm{~g} / \mathrm{ml}$ sodium chloride, and bitter taste $(0.006 / 0.0024$ $/ 0.0009 / 0.0004 \mathrm{~g} / \mathrm{ml}$ quinin-hydrochloride), respectively. Two strips were blanks (no tastants). Testing was started with the lowest concentrations and the order of presentation was only reproducible by the examiner. In patients tested after ear surgeries, taste strips were placed on the corresponding anterior tongue side. All other participants placed taste strips central on the anterior two-thirds of the tongue and after presentation of each taste strip, participants took a sip of tap water. Participants were asked to respond according to a list of five possible answers (sweet, sour, salty, bitter, no taste) in case of non-forcedchoice procedure (nfc) and to a list of four possible answers (sweet, sour, salty, bitter) in case of forced-choice procedure (fc). As previously published ${ }^{(18)}$, each correct answer counted one point, incorrect identified taste qualities yielded no points. 
Table 1. Taste strips answers in percent $(n=102)$.

\begin{tabular}{|c|c|c|c|c|c|c|}
\hline $\mathrm{fc} / \mathrm{nfc}$ & correct & no taste & sweet & sour & salty & bitter \\
\hline sweet 1 & 98.0 / 100 & / & & / & $2.0 /$ & / \\
\hline sweet 2 & 96.1 / 97.1 & / 1.0 & & $1.0 /$ & $2.0 / 1.0$ & $1.0 / 1.0$ \\
\hline sweet 3 & 90.2 / 84.3 & / 3.9 & & $2.0 / 3.9$ & $2.9 / 2.0$ & $4.9 / 5.9$ \\
\hline sweet 4 & 77.5 / 78.4 & / 7.8 & & $7.8 / 2.0$ & $6.9 / 2.9$ & $7.8 / 8.8$ \\
\hline sour 1 & 87.3 / 91.2 & / & / & & $7.8 / 5.9$ & $4.9 / 2.9$ \\
\hline sour 2 & 80.4 / 77.5 & $/ 2.0$ & $1.0 /$ & & $14.7 / 17.6$ & $3.9 / 2.9$ \\
\hline sour 3 & $52.0 / 52.0$ & / 15.7 & $4.9 / 2.0$ & & $31.4 / 22.5$ & $3.9 / 2.9$ \\
\hline sour 4 & 14.7 / 7.8 & / 69.6 & $12.7 / 3.9$ & & $33.3 / 7.8$ & $39.2 / 10.8$ \\
\hline salty 1 & 92.2 / 89.2 & / & $1.0 / 2.0$ & $4.9 / 5.9$ & & $2.0 / 1.0$ \\
\hline salty 2 & 82.4 / 87.3 & / 2.0 & $2.0 /$ & 13.7 / 9.8 & & $2.0 / 1.0$ \\
\hline salty 3 & 67.6 / 70.6 & / 3.9 & 6.9 / 1.0 & $20.6 / 16.7$ & & $4.9 / 5.9$ \\
\hline salty 4 & 70.6 / 60.8 & / 12.7 & $3.9 / 2.0$ & $20.6 / 18.6$ & & $4.9 / 5.9$ \\
\hline bitter 1 & 97.1 / 94.1 & / 2.9 & / & $1.0 / 2.0$ & $2.0 / 1.0$ & \\
\hline bitter 2 & $85.3 / 85.3$ & / 6.9 & $2.9 / 2.9$ & $5.9 / 4.9$ & $5.9 /$ & \\
\hline bitter 3 & 69.6 / 65.7 & / 19.6 & $5.9 / 5.9$ & $14.7 / 4.9$ & $9.8 / 3.9$ & \\
\hline bitter 4 & $67.6 / 42.2$ & / 39.2 & $10.8 / 6.9$ & $7.8 / 4.9$ & $13.7 / 6.9$ & \\
\hline blank 1 & & / 75.5 & $19.6 / 3.9$ & $26.5 / 3.9$ & $16.7 / 5.9$ & $37.3 / 10.8$ \\
\hline blank 2 & & / 78.4 & $19.6 / 2.9$ & $16.7 / 6.9$ & $22.5 / 4.9$ & 41.2 / 6.9 \\
\hline
\end{tabular}

fc, forced-choice; nfc, non-forced-choice; Highest concentration (1) to lowest concentration (4).

Each testing modality added up to a comparable score with a maximum score of 16 (blanks yielded no points). The cut-off for hypogeusia is less than nine points, corresponding to the 10th percentile, as proposed in the initial taste strips study by Mueller et al. and reproduced with a larger sample size by Landis et al. using a forced choice paradigm ${ }^{(8,27)}$. The collected taste scores assessed by both procedures ( $\mathrm{fc}$ and $\mathrm{nfc}$ ) represented the main objective parameter in this study.

\section{Statistical analysis}

IBM SPSS 25.0 (Chicago, IL, USA) was used for statistical analysis. Individual parameters and taste scores were transferred to a multivariate analysis of variance for repeated measures (general linear model, GLM) using taste quality (4 qualities) and method (fc and $\mathrm{nfc}$ choice) as within subject factors. Testing sequence (1st nfc or 1st fc) and gender were included as between-subject factors. Body mass index (BMI) and age served as covariates. In case of missing sphericity Greenhouse-Geisser corrected p-values were used. In order to test specific differences at the exploratory level, Wilcoxon-Test (for within subject variables) and Mann-Whitney U-Test (for between subjects' variables) were used as post hoc tests. Correlational analyses were performed using Spearman's rho ( $\rho$ ). Taste score data are presented as boxand-whisker plots using quartiles (interquartile range: Q0.25, Q0.75; boxes), medians (Q0.5; line), and the 5th/95th percentiles (Q0.5 and Q0.95; whiskers). Parametric data are presented as mean and standard deviation of mean (SD), as indicated. The alpha level was set at 0.05. GraphPrism 7.0 (GraphPad Software, La Jolla, California, USA) was used to visualize data.

\section{Results}

Healthy participants

This group showed median scores of 13 (interquartile range,

11-14) for fc and 12 (interquartile range, 10-14) for nfc. Although subjectively unimpaired, 11 participants scored less than 9 points on TS (10 male, 1 female), indicating hypoguesia, of which 6 failed to reach the cut-off in both procedures ( $\mathrm{fc}$ and $\mathrm{nfc}$ ). The analysis of variance revealed an interaction of factor quality and gender $(p=0.038, d f=1,24)$. Post hoc test showed a gender effect on bitter taste only: males scored significantly lower than females on overall bitter taste scores $(p=0.016$, Mann-Whitney-U-Test). Table 1 shows hit rates of all qualities and concentrations in this group of 102 participants. Healthy participants' mean body-mass-index (BMI \pm SD) was 24.1 \pm 3.9 and mean SAS, SAT and SAH were 7.2 $\pm 2.0,7.2 \pm 1.5$ and $3.1 \pm 2.4$. Thirty-four (33.3\%) subjects claimed they were smoker. Age negatively affected test results $(\rho 102=-0.52, p<0.001)$ : TS results were significantly lower the older the participant. Older (and male) participants presented with a higher BMI. Subjective assessment of smell and taste did not significantly correlate with TS scores ( $p>0.05$, Spearman`s $\rho$ ). Also, SAH did not seem to effect testing performance ( $p>0.05$, Spearman`s $\rho$ ). In healthy subjects, the lowest sour strip was named correct only in $14.7 \%$ (fc) and $7.8 \%$ (nfc). In nfc the two blank strips were correctly named blank in $75.5 \%$ and $78.4 \%$ cases, respectively. In wrong blank cases bitter was named the most instead of blank (10.8\% and $6.9 \%$, respectively). In fc procedure bitter was the most chosen quality when blanks were administered $(37.3 \%$ and $41.2 \%$, respectively) (Table 1 ). 
Table 2. Results of participants tested in a forced (fc) and a non-forced (nfc) choice testing procedure and reasons for possible taste dysfunction.

\begin{tabular}{|c|c|c|c|c|c|c|}
\hline & $\mathbf{n}$ & Day & $\begin{array}{c}\text { fc } \\
\text { Mean }\end{array}$ & SD & $\begin{array}{c}\text { nfc } \\
\text { Mean }\end{array}$ & SD \\
\hline Round window sealing tympanotomy & 3 & 1 & 7.3 & 3.4 & 5.3 & 3.4 \\
\hline Stapes Surgery & 7 & $1-2$ & 7.9 & 3.4 & 6.4 & 2.7 \\
\hline History of multiple cholesteatoma surgeries & 2 & $55 / 100$ & 7.5 & 4.5 & 8.5 & 2.5 \\
\hline Cholesteatoma surgery & 2 & 2 & 9.5 & 1.5 & 10 & 1 \\
\hline Tympanoplasty with CTN manipulation & 3 & 1 & 9.7 & 3.3 & 7.0 & 0.8 \\
\hline Ossiculoplasty with severed CTN & 1 & 1 & 7 & & 9 & \\
\hline Tonsillectomy & 10 & $2-3$ & 12.6 & 2.2 & 12.9 & 1.6 \\
\hline Self-reported hypogeusia & 2 & $30 / 90$ & 12.5 & 2.1 & & 2.8 \\
\hline All & 30 & & 9.9 & 3.7 & 9.3 & 3.6 \\
\hline
\end{tabular}

CTN, Chorda tympani nerve; "Day" indicates how many days after surgeries or onset of symptoms participants were tested.

\section{Patients with potential taste dysfunction}

Eighteen patients were tested after ear surgeries. Ten patients were tested after tonsillectomies. Two patients reported taste dysfunction. Patients' characteristics and mean scores of both modalities are shown in Table 2. Patients' mean BMI was 24.5 \pm 4.6 and mean SAS, SAT and SAH were $7.8 \pm 2.3,6.9 \pm 2.4$ and $2.9 \pm 3.2$.

Two out of 3 patients after round window sealing through a tympanotomy approach subjectively (SAT 3 and 7, respectively) and psychophysically experienced a decrease in taste function on the operated side on the first day after surgery. Six out of 7 patients after stapes surgery yielded below 9 points in at least one modality. Scores in stapes patients were lower in $\mathrm{nfc}$, although due to small sample size the difference did not reach significance ( $p>0.05)$.

Amongst the investigated tonsillectomy patients median tongue testing in both modalities revealed no scores below the suggested cut-off for hypogeusia. Two of these patients showed scores of 9 in at least one modality, hence at the edge to hypogeusia. Subjective impairment however was not reported among these 2 patients with SAT scores as high as 8 and 10, respectively.

In tested patients $(n=30)$ there was a clear interaction method (fc vs. $n f c)$ by quality and age $(p=0.001 ; d f=3.22)$. Post hoc tests showed that bitter taste perception differed significantly in $\mathrm{fc}$ (median, 3; interquartile range, $2-4$ ) and nfc (median, 3 ; interquartile range, 1-4) modalities ( $p=0.016$, Wilcoxon matched-pairs ranked sign test), while other qualities showed no significant difference $(p>0.05)$ (Figure 1).

\section{All participants}

When gathering all tested subjects $(n=132)$, there was again an interaction method ( $f c$ vs. $n f c)$ by quality and sequence ( $p=$
$0.039 ; \mathrm{df}=3,124)$. Descriptive statistics of all participants by gender, including TS results are presented in Table 3. As seen in Figure 2, bitter taste perception differed significantly in $\mathrm{fc}$ and $n f c$ ( $p<0.0001$, Wilcoxon matched-pairs ranked sign test), while other qualities showed no significant difference $(p>0.05)$. Irrespective of testing sequence, there was also an interaction quality by gender $(p<0.001, d f=3,124)$.

\section{Test sequence}

In the overall group of 132 participants there was interaction method by quality by sequence $(p=0.039, d f=3,124)$. The bitter score in nfc was significant lower in the sequence fc $1 \mathrm{st}$ - subgroup ( $p=0.042$, Wilcoxon matched-pairs ranked sign test), but this effect was more visible in the sequence nfc 1 st - subgroup ( $p<0.001$ for bitter taste, Wilcoxon matched-pairs ranked sign test) and hence indicating in nfc 1st - subgroup "no taste" was chosen more often for bitter taste strips.

Assessing test-retest reliability, scores of the two procedures of all participants correlated significantly $(\rho 132=0.76 ; p<0.0001)$, as expected due to short testing intervals and exceeding results from previous authors ${ }^{(8,25)}$.

\section{Discussion}

As a major finding of the present investigation, applied strategy (forced or non-forced-choice paradigm) affected bitter taste strips scores in overall tested participants, as well as in a smaller group of 30 tested patients.

From an observer point of view, the question of providing "no taste" as an answer option in gustatory testing seems to be trivial. Nonetheless, the emerging interest for gustatory function, and taste buds as a possible piece of the puzzle to understanding other diseases, underlines the necessity for standardized taste tests. 


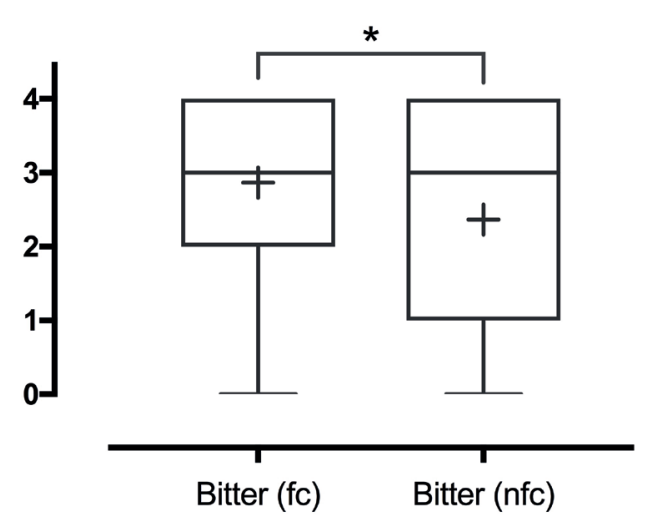

Figure 1. Bitter taste scores per testing procedure in a group of patients tested after potentially taste harming surgeries (e.g., stapes surgeries, tonsillectomies) or with subjective taste dysfunction; fc, forced-choice; nfc, non-forced-choice; + indicating the mean scores; ${ }^{*}$ Asterisk indicating significant differences between bitter scores ( $p=0.016$, Wilcoxon matched-pairs ranked sign test).

The advantage of the nfc paradigm, as it was initially proposed ${ }^{(8)}$, is the possibility to assess taste detection and identification in subjects separately. This means if a taste strip is assigned to a wrong tastant by the investigated subject, the examiner may conclude this specific taste quality was not recognized correctly, but a stimulus was detected. In a fc paradigm it cannot be determined if a correct answer is given due to correct recognition, due to detection of a stimulus that was not correctly recognized or due to mere guessing. In fc however, scores close to zero are unlikely and subjects, being obliged to choose from sweet, sour, salty and bitter, might be more ambitious to recognize it and therefore, as Landis et al. argued, $\mathrm{fc}$ was better able to detect remnants of gustatory function ${ }^{(27)}$. Bartoshuk discussed subject's "conservativeness" having an influence on decision making in taste tests: less conservative subjects may decide even if they are less certain ${ }^{(28)}$. Within our cohort, subjects frequently reported to feel more comfortable having the option to choose "no taste". Eventually both, fc and nfc procedures, seem to be applicable. Table 4 provides an overview to discussed advantages and disadvantages of chosen paradigms in gustatory testing. How relevant is forced-choice and non-forced-choice testing in diagnosing olfactory disorders? Describing olfactory stimuli seems to be of same difficulty such us describing human faces despite recognizing them ${ }^{(3)}$. Providing verbal cues to a smell tremendously helps in naming an odour. For this reason and also due to multiple existing olfactory stimuli, there is a presumable bias if no answer is given. Therefore, traditionally olfactory testing is performed in a forced-choice manner ${ }^{(29,30)}$. Additionally, non-forced-choice olfactory tests have been developed, like the validated 5-item sniffin' sticks test, which utilizes only 5 different odours and various descriptors including "no odour" to screen for anosmia ${ }^{(31)}$. However, also with regard to detecting malinge-
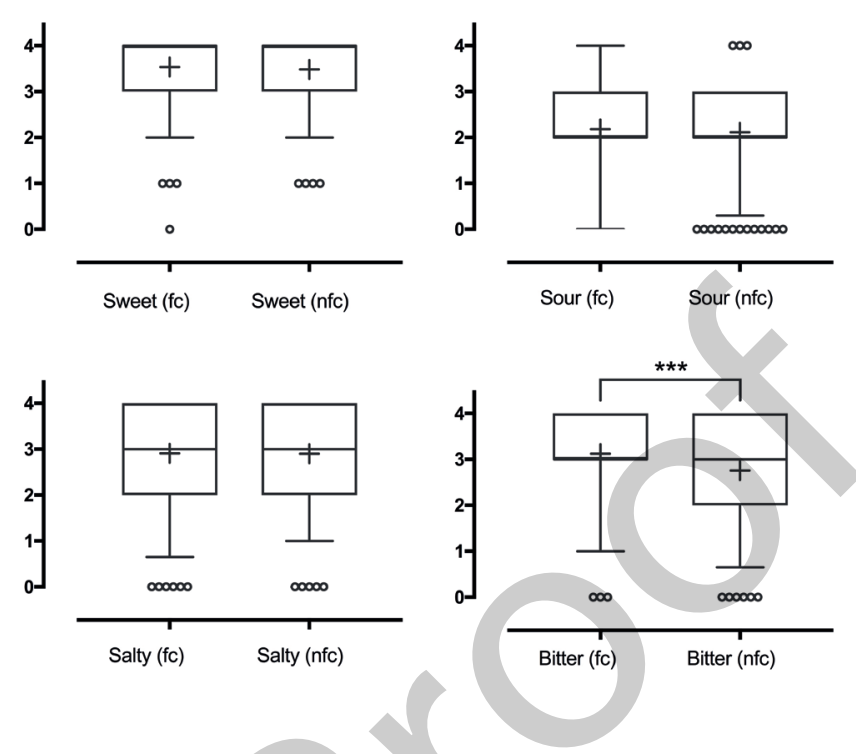

Figure 2. Comparative illustration of all subjects` scores per testing procedure of each quality ( $n=132)$. $f c$, forced-choice; $n f c$, non-forced-choice; + indicating the mean scores; ${ }^{* * *}$ Asterisk indicating significant differences between bitter scores ( $p<0.0001$, Wilcoxon matched-pairs ranked sign test).

ring subjects ${ }^{(32)}$, forced-choice testing is recommended in more elaborate olfactory test situations with a larger number of items. But it has also to be kept in mind that a trained (informed) person will be able to counteract this forced choice test procedure. Therefore, a forced choice test procedure is not able to unmask all malingering subjects.

In gustatory testing only four (five including umami ${ }^{(19)}$ ) qualities can be detected. Due to the small number of taste qualities and according small numbers of substances, the TS test has been designed as a semi-quantitative test with four different concentrations presented from the lowest to the highest concentrations (and an extension with six concentrations for each taste quality $\left.{ }^{(23)}\right)$. The lowest concentration was chosen to be detected by a maximum of fifty percent of healthy subjects ${ }^{(8)}$.

In the present investigation, more than one third of subjects were not able to detect the lowest bitter concentration in nfc answering "no taste". On the other hand, blanks were often confused with bitter taste strips or named bitter when administered in fc. In concordance to previous findings, overall woman and younger participants scored significantly better ${ }^{(23,27,33)}$, which was the case for both procedures (fc and $\mathrm{nfc}$ ).

To investigate for reproducibility of possible differences in health and disease, we tested patients with potential impairments of gustatory function after ear surgeries, after tonsillectomies, and patients with subjective hypogeusia. In stapes surgery several studies reported transient gustatory impaired and also feeling of numbness on the corresponding tongue side due to mild to severe chorda tympani nerve (CTN) manipulation ${ }^{(22,34,35)}$. 
Table 3. All participants' characteristics and taste strips test results by gender.

\begin{tabular}{|ccccc} 
& \multicolumn{2}{c}{ female $(\mathbf{n = 7 0})$} & \multicolumn{2}{c}{ male $(\mathbf{n = 6 2})$} \\
& Mean & SD & Mean & SD \\
\hline Age & 37.8 & 17.4 & 40.6 & 18.4 \\
\hline BMI & 22.6 & 3.5 & 25.7 & 4.2 \\
\hline SAS & 7.5 & 1.9 & 7.2 & 2.3 \\
\hline SAT & 7.2 & 1.7 & 7.0 & 1.8 \\
\hline SAH & 2.5 & 2.3 & 3.7 & 2.8 \\
\hline fC & 12.2 & 2.8 & 11.3 & 3.1 \\
\hline nfC & 11.8 & 2.8 & 10.6 & 3.1 \\
\hline
\end{tabular}

BMI, Body-mass-index; fc, forced-choice; nfc, non-forced-choice; SAH, subjective assessment of hunger; SAS, subjective assessment of smell; SAT, subjective assessment of taste.
Table 4. Overview on possible advantages and disadvantages of forcedand non-forced-choice paradigms in psychophysically testing of gustatory function.

\begin{tabular}{|c|c|c|}
\hline & Advantage & Disadvantage \\
\hline $\begin{array}{l}\text { Forced- } \\
\text { choice }\end{array}$ & $\begin{array}{l}\text { No scores close to zero } \\
\text { Detection of function } \\
\text { "remnants" } \\
\text { No real difference if per- } \\
\text { son is decisive }\end{array}$ & $\begin{array}{l}\text { Higher scores by sheer } \\
\text { guessing } \\
\text { No valuable use of blanks } \\
\text { Scores }>0 \text { even in ageusia } \\
\text { (but diagnosis of ageusia } \\
\text { impossible) }\end{array}$ \\
\hline $\begin{array}{l}\text { Non-for- } \\
\text { ced-choice }\end{array}$ & $\begin{array}{l}\text { More comfortable test } \\
\text { setting } \\
\text { Rational integration of } \\
\text { blanks } \\
\text { Differentiation between } \\
\text { stimuli detection and } \\
\text { identification }\end{array}$ & $\begin{array}{l}\text { Personality bias } \\
\text { Malingerer may perform } \\
\text { worse } \\
\text { More data on forced- } \\
\text { choice available so far }\end{array}$ \\
\hline
\end{tabular}

"Blanks" refer to taste strips without tastant.

participants reported the second taste testing cycle was easier, possibly because stimuli had already been experienced in high concentrations. In case $\mathrm{nfc}$ was applied first, missing experience on taste strip concentrations may have contributed to the mentioned findings concerning test sequence and "no taste" was chosen more often. In clinical routine however, testing is not performed twice on a regular basis. Researchers however may have to consider "learning effects" in bitter taste testing using taste strips depending on paradigm.

Bitter taste perception seems to be more complex and challenging than other qualities. Above mentioned behavioural modifications influence bitter taste scores in a relevant way depending on paradigm. Alterations in bitter taste perception in patients with CRS have been reported by Wolf et al. using a nfC and Othieno et al. using a fc paradigm ${ }^{(16,17)}$. Given the present study's findings, paradigm selection may interfere with bitter taste test scores, which has to be taken into account when comparing studies on bitter taste perception. In respect to further investigations, shown effect may be more pronounced when applying lower concentrations as in the extended version of the taste strips test ${ }^{(23)}$.

\section{Conclusion}

Taste strips are valid to be used in a forced and a non-forced choice paradigm, with bitter taste showing slightly higher scores in forced-choice testing. Differences between test procedures may also be found in smaller groups and more often in male subjects. Future investigators with focus on bitter taste perception should be particularly cautious when comparing results in regard to testing procedure.

\section{Acknowledgements}

This study obtained no third-party funding. 


\section{Authorship contribution}

GB: collection of data, analysis of results, write-up of manuscript, critical review of all contents; AP: collection of data, critical review of all contents; CAM: concept of study, critical review of all contents; BR: concept of study, analysis of results, critical review of all contents.

\section{Conflict of interest}

All authors state to have no conflict of interest.

\section{References}

1. Spence C. Multisensory flavour perception. Cell. 2015;161(1):24-35.

2. Breslin PAS. An evolutionary perspective on food and human taste. Curr Biol. 2013;23(9):409-18.

3. Shepherd GM. Smell images and the flavour system in the human brain. Nature. 2006;444:316-321.

4. Deems DA, Doty RL, Settle RG, et al. Smell and Taste Disorders, A Study of 750 Patients From the University of Pennsylvania Smell and Taste Center. Arch Otolaryngol Head Neck Surg. 1991;117(5):519-28.

5. Bojanowski V, Hummel T. Retronasal perception of odors. Physiol Behav. 2012;107(4):484-7.

6. Landis BN, Stow NW, Lacroix JS, Hugentobler M, Hummel T. Olfactory disorders: the patients' view. Rhinology. 2009;47(4):1-6.

7. Soter A, Kim J, Jackman A, Tourbier I, Kaul A, Doty RL. Accuracy of self-report in detecting taste dysfunction. Laryngoscope Investig Otolaryngol. 2008;118(4):611-7.

8. Mueller C, Kallert S, Renner B, et al. Quantitative assessment of gustatory function in a clinical context using impregnated "taste strips". Rhinology. 2003;41(1):2-6.

9. Han P, Georgi M, Cuevas M, Haehner A, Gudziol V, Hummel T. Decreased Electrogustometric Taste Sensitivity in Patients with Acquired Olfactory Dysfunction. Rhinology. 2018;56(2):158-65.

10. Schuster B, lannilli E, Gudziol V, Landis BN. Gustatory testing for clinicians. B-ENT. 2009;13:109-13.

11. Hummel T, Whitcroft $K$, Andrews $P$, et al Position paper on olfactory dysfunction. Rhinol Suppl. 2017;54(26):1-30.

12. Yamamoto K, Ishimaru Y. Oral and extraoral taste perception. Semin Cell Dev Biol. 2013;24(3):240-6.

13. Lee RJ, Cohen NA. Bitter and sweet taste receptors in the respiratory epithelium in health and disease. J Mol Med. 2014;92(12):1235-44.

14. Lee RJ, Cohen NA. The emerging role of the bitter taste receptor T2R38 in upper respiratory infection and chronic rhinosinusitis. Am J Rhinol Allergy. 2013;27(4):283-6.

15. Rom DI, Christensen JM, Alvarado R, Sacks R, Harvey RJ. The impact of bitter taste receptor genetics on culturable bacteria in chronic rhinosinusitis. Rhinology. 2017;55(1):90-4.

16. Wolf A, Renner B, Tomazic PV, Mueller CA Gustatory Function in Patients With Chronic Rhinosinusitis. Ann Otol Rhinol Laryngol. 2018;127(4):229-34
17. Othieno F, Schlosser RJ, Rowan NR, et al. Taste impairment in chronic rhinosinusitis. Int Forum Allergy Rhinol. 2018;23(1):139.

18. Wolf A, Varga L, Wittibschlager $L$, Renner B, Mueller CA. A self-administered test of taste function using "Taste Strips". Int Forum Allergy Rhinol. 2016;6(4):362-6.

19. Mueller CA, Pintscher K, Renner B. Clinical test of gustatory function including umami taste. Ann Otol Rhinol Laryngol. 2011;120(6):358-62.

20. Mueller CA, Khatib S, Landis BN, Temmel AFP, Hummel T. Gustatory function after tonsillectomy. Arch Otolaryngol Head Neck Surg. 2007;133(7):668-71.

21. Mueller CA, Khatib S, Temmel AFP Baumgartner W-D, Hummel T. Effects of cochlear implantation on gustatory function. Ann Otol Rhinol Laryngol. 2007;116(7):498-501.

22. Mueller CA, Khatib S, Naka A, Temmel AFP, Hummel T. Clinical Assessment of Gustatory Function before and after Middle Ear Surgery: A Prospective Study with a TwoYear Follow-up Period. Ann Otol Rhinol Laryngol. 2008;117(10):769-73.

23. Wolf A, Illini O, Uy D, Renner B, Mueller CA. A new extension to the Taste Strips test. Rhinology. 2016;54(1):45-50.

24. Walliczek-Dworschak U, Pellegrino R, Taube $F$, et al. Chemosensory function before and after multimodal treatment in chronic rhinosinusitis patients. Laryngoscope. 2018;128(3):86-90.

25. Fjaeldstad A, Niklassen AS, Fernandes HM. Re-Test Reliability of Gustatory Testing and Introduction of the Sensitive Taste-DropTest. Chem Senses. 2018;43(5):341-6.

26. Walliczek-Dworschak U, Knauer CM, Murbe D, Mainka A, Hummel T. Analysis of taste function in patients before and after cochlear implant surgery. Rhinology. 2018;56(2):149-54.

27. Landis $B N$, Welge-Luessen $A$, Brämerson A, et al. "Taste Strips" - a rapid, lateralized, gustatory bedside identification test based on impregnated filter papers. J Neurol. 2009;256(2):242-8.

28. Bartoshuk LM. The psychophysics of taste. Am J Clin Nutr. 1978;1;31(6):1068-77.

29. Doty RL. Office procedures for quantitative assessment of olfactory function. Am J Rhinol. 2007;21(4):460-73.

30. Hummel T, Kobal G, Gudziol H, Mackay-Sim A. Normative data for the "Sniffin' Sticks" including tests of odor identification, odor discrimination, and olfactory thresholds: an upgrade based on a group of more than 3,000 subjects. Eur Arch Otorhinolaryngol. 2007;264(3):237-43.
31. Mueller C, Renner B. A new procedure for the short screening of olfactory function using five items from the "Sniffin' Sticks" identification test kit. Am । Rhinol. 2006;20(1):113-6.

32. Doty RL. Measurement of chemosensory function. World J Otorhinolaryngol Head Neck Surg. 2018;4(1):11-28.

33. Barragán R, Coltell O, Portolés O, et al. Bitter, Sweet, Salty, Sour and Umami Taste Perception Decreases with Age: SexSpecific Analysis, Modulation by Genetic Variants and Taste-Preference Associations in 18 to 80 Year-Old Subjects. Nutrients. 2018;10(10):1539.

34. Maeda E, Katsura H, Nin T, SakaguchiFukunaga A, Mishiro Y, Sakagami M. Change of somatosensory function of the tongue caused by chorda tympani nerve disorder after stapes surgery. Laryngoscope. 2018;128(3):701-6.

35. Guder E, Böttcher A, Pau HW, Just T. Taste function after stapes surgery. Auris Nasus Larynx. 2012;39(6):562-6.

36. Windfuhr JP, Sack F, Sesterhenn AM, Landis BN, Chen Y-S. Post-tonsillectomy taste disorders. Eur Arch Otorhinolaryngol. 2010;267(2):289-93.

37. Temmel AFP, Quint C, SchickingerFischer B, Klimek L, Stoller E, Hummel T. Characteristics of olfactory disorders in relation to major causes of olfactory loss. Arch Otolaryngol Head Neck Surg. 2002;128(6):635-41.

38. Kobayashi C, Kennedy LM, Halpern BP. Experience-induced changes in taste identification of monosodium glutamate (MSG) are reversible. Chem Senses. 2006;31(4):301-6.

39. Gonzalez KM, Peo C, Livdahl T, Kennedy LM. Experience-induced changes in sugar taste discrimination. Chem Senses. 2008;33(2):173-9.

\section{Christian A. Mueller, MD}

Department of Otorhinolaryngology and Head and Neck Surgery

Medical University of Vienna

Währinger Gürtel 18-20

1090 Vienna

Austria

Tel: +43-1-40-400 33340

E-mail: christian.a.mueller @meduniwien.ac.at 